\title{
Therapeutic regulation of VE-cadherin with a novel oligonucleotide drug for diabetic eye complications using retinopathy mouse models
}

\author{
Ka Ka Ting ${ }^{1} \cdot$ Yang Zhao ${ }^{1}$. Weiyong Shen ${ }^{2} \cdot$ Paul Coleman $^{1} \cdot$ Michelle Yam ${ }^{2} \cdot$ Tailoi Chan-Ling $^{3} \cdot$ Jia Li $^{1}$ • \\ Thorleif Moller ${ }^{4}$ • Mark Gillies ${ }^{2} \cdot$ Mathew A. Vadas ${ }^{1}$ • Jennifer R. Gamble ${ }^{1}$
}

Received: 9 March 2018 / Accepted: 3 October 2018 / Published online: 15 November 2018

(C) Springer-Verlag GmbH Germany, part of Springer Nature 2018

\begin{abstract}
Aims/hypothesis A major feature of diabetic retinopathy is breakdown of the blood-retinal barrier, resulting in macular oedema. We have developed a novel oligonucleotide-based drug, CD5-2, that specifically increases expression of the key junctional protein involved in barrier integrity in endothelial cells, vascular-endothelial-specific cadherin (VE-cadherin). CD5-2 prevents the mRNA silencing by the pro-angiogenic microRNA, miR-27a. CD5-2 was evaluated in animal models of ocular neovascularisation and vascular leak to determine its potential efficacy for diabetic retinopathy.

Methods CD5-2 was tested in three mouse models of retinal dysfunction: conditional Müller cell depletion, streptozotocininduced diabetes and oxygen-induced retinopathy. Vascular permeability in the Müller cell-knockout model was assessed by fluorescein angiography. The Evans Blue leakage method was used to determine vascular permeability in streptozotocin- and oxygen-induced retinopathy models. The effects of CD5-2 on retinal neovascularisation, inter-endothelial junctions and pericyte coverage in streptozotocin- and oxygen-induced retinopathy models were determined by staining for isolectin-B4, VE-cadherin and neural/glial antigen 2 (NG2). Blockmir CD5-2 localisation in diseased retina was determined using fluorescent in situ hybridisation. The effects of CD5-2 on VE-cadherin expression and in diabetic retinopathy-associated pathways, such as the transforming growth factor beta (TGF- $\beta$ ) and wingless/integrated (WNT) pathway, were confirmed using western blot of lysates from HUVECs, a mouse brain endothelial cell line and a VE-cadherin null mouse endothelial cell line.

Results CD5-2 penetrated the vasculature of the eye in the oxygen-induced retinopathy model. Treatment of diseased mice with CD5-2 resulted in reduced vascular leak in all three animal models, enhanced expression of VE-cadherin in the microvessels of the eye and improved pericyte coverage of the retinal vasculature in streptozotocin-induced diabetic models and oxygen-induced retinopathy models. Further, CD5-2 reduced the activation of retinal microglial cells in the streptozotocin-induced diabetic model. The positive effects of CD5-2 seen in vivo were further confirmed in vitro by increased protein expression of VEcadherin, SMAD2/3 activity, and platelet-derived growth factor B (PDGF-B).

Conclusions/interpretation CD5-2 has therapeutic potential for individuals with vascular-leak-associated retinal diseases based on its ease of delivery and its ability to reverse vascular dysfunction and inflammatory aspects in three animal models of retinopathy.
\end{abstract}

Yang Zhao and Weiyong Shen contributed equally to this study.

Electronic supplementary material The online version of this article (https://doi.org/10.1007/s00125-018-4770-4) contains peer-reviewed but unedited supplementary material, which is available to authorised users.

Jennifer R. Gamble

j.gamble@centenary.org.au

Mathew A. Vadas

m.vadas@centenary.org.au

1 Centre for the Endothelium Vascular Biology Program Centenary Institute, The University of Sydney, Locked bag \#6,

Newtown, NSW 2042, Australia
2 Save Sight Institute, The University of Sydney, Sydney, NSW, Australia

3 Discipline of Anatomy and Histology, School of Medical Sciences Bosch Institute, The University of Sydney, Sydney, NSW, Australia

4 Ranger Biotechnologies A/S, Årslev, Denmark 


\section{Research in context}

\section{What is already known about this subject?}

- Diabetic retinopathy pathogenesis involves a cascade of events leading to retinal lesions, including pericyte loss, blood-retinal barrier breakdown, pre-retinal neovascularisation, inflammation, glial activation and neuronal apoptosis

- Current treatments include laser treatment, intravitreal injection of anti-vascular endothelial growth factor (VEGF) or steroids, and surgery

\section{What is the key question?}

- Can targeting vascular-endothelial-specific cadherin (VE-cadherin) in endothelial cells be used as a therapeutic strategy for diabetic retinopathy?

\section{What are the new findings?}

- A novel drug, CD5-2, which localises to vascular endothelial cells in the mouse eye, increases endogenous VE-cadherin expression in diabetic and oxygen-induced retinopathy models

- $\quad$ CD5-2 reduces vascular leakage, improves pericyte coverage and maintains VE-cadherin integrity in the retinal vasculature in diabetic and proliferative models of retinopathy

- CD5-2 inhibits microglial activation in diabetic mice

How might this impact on clinical practice in the foreseeable future?

- $\mathrm{CD} 5-2$ may be an alternative strategy for the treatment of diabetic retinopathy

Keywords Blood-retinal barrier - Diabetic retinopathy $\cdot$ Microglia $\cdot$ MicroRNA $\cdot$ Müller cells $\cdot$ Neovascularisation · Oligonucleotide $\cdot$ Pericytes $\cdot$ VE-cadherin

$\begin{array}{ll}\text { Abbreviations } & \\ \text { BBB } & \text { Blood-brain barrier } \\ \text { BRB } & \text { Blood-retinal barrier } \\ \text { DMO } & \text { Diabetic macular oedema } \\ \text { eNOS } & \text { Endothelial nitric oxide synthase } \\ \text { GAPDH } & \text { Glyceraldehyde-3-phosphate dehydrogenase } \\ \text { GCL } & \text { Ganglion cell layer } \\ \text { INL } & \text { Inner nuclear layer } \\ \text { KO } & \text { Knockout } \\ \text { LRP6 } & \text { LDL receptor-related protein } 6 \\ \text { miRNA } & \text { MicroRNA } \\ \text { NG2 } & \text { Neural/glial antigen 2 } \\ \text { OIR } & \text { Oxygen-induced retinopathy } \\ \text { ONL } & \text { Outer nuclear layer } \\ \text { P7/12/14/17 } & \text { 7/12/14/17 days postnatal } \\ \text { PDGF-B } & \text { Platelet-derived growth factor B } \\ \text { PDGFR- } \beta & \text { Platelet-derived growth factor receptor } \beta \\ \text { PDR } & \text { Proliferative diabetic retinopathy } \\ \text { SMAD } & \text { Smad family member } \\ \text { STZ } & \text { Streptozotocin } \\ \text { VE-cadherin } & \text { Vascular-endothelial-specific cadherin } \\ \text { VEC-null } & \text { VE-cadherin null } \\ \text { VEGF } & \text { Vascular endothelial growth factor } \\ \text { WNT } & \text { Wingless/integrated } \\ \end{array}$

\section{Introduction}

Diabetic retinopathy is a microvascular complication of diabetes mellitus and a major cause of visual impairment and blindness among people with diabetes. The development of diabetic retinopathy affects the retina and can be classified into early-stage non-proliferative diabetic retinopathy (NPDR) and late-stage proliferative diabetic retinopathy (PDR) [1]. In addition, individuals with diabetic retinopathy can develop diabetic macular oedema (DMO), another contributing factor to vision loss [1].

The central component of diabetic retinopathy pathology involves breakdown of the blood-retinal barrier (BRB), which is normally impermeable and highly selective for molecules moving in and out of the retina [2]. The integrity of the inner barrier depends on the retinal vascular unit, which is composed of capillary endothelial cells interconnected by adherens and tight junctions and surrounded by pericytes which, in turn, are supported by astrocyte and Müller glial cells [2].

Breakdown of the BRB results in plasma leak, culminating in the development of DMO, intraretinal haemorrhages and hard exudates. One of the earliest features of breakdown of the $\mathrm{BRB}$ in diabetic retinopathy is the loss of pericytes [3], with 
a
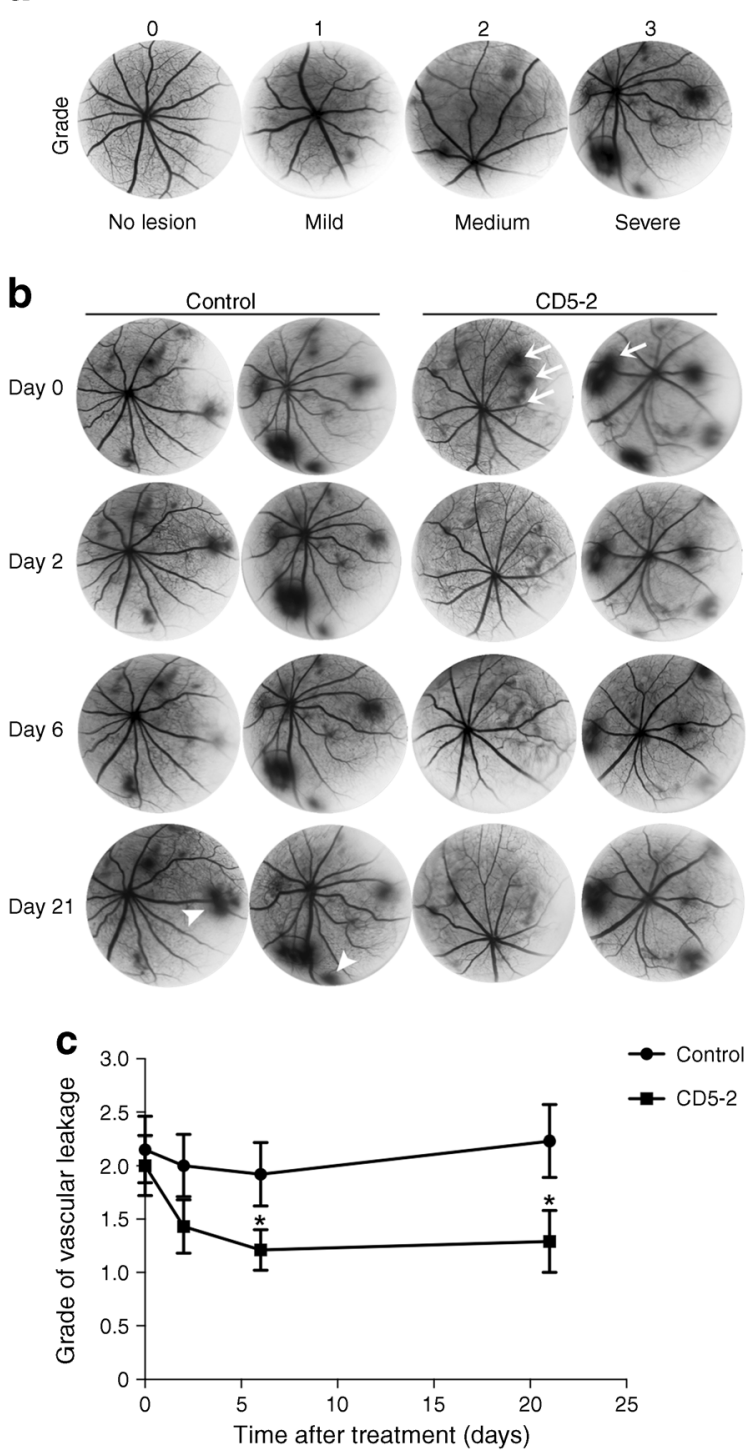

Fig. 1 CD5-2 reduces focal vascular leakage in conditional Müller cell$\mathrm{KO}$ mice. (a) Scales used for grading vascular leakage revealed by fluorescein angiography: grade 0 , normal pattern of fluorescein filling with no vascular leakage; grade 1, readily identifiable lesions showing diffuse leakage but without intense focal vascular leakage; grade 2, intense focal vascular leakage appears but the lesion number is $\leq 3$; grade 3, 4-6 lesions with intense focal vascular leakage; and grade $4,>6$ lesions with intense focal vascular leakage (not shown). (b) Representative angiography of vascular leakage before (day 0 ) and after treatment with control or CD5-2 (days 2-21). White arrows show established lesions in CD5-2-treated mice at day 0 . Arrowheads show enlarged lesions in control-treated group at day 21. (c) Grade of vascular leakage in Müller cell-KO mice over the course of 3 weeks. * $p<0.05$ control vs CD5-2-treated mice by unpaired Student's $t$ test. Control-treated mice, $n=3$; CD5-2-treated mice, $n=4$

subsequent focal destabilisation of the vessel wall. This occurs as the endothelial junctions weaken, mediated by loss of localisation of the endothelial-cell-specific adherens junctional protein, vascular-endothelial-specific cadherin (VEcadherin), and decreases in the expression of claudin-5 and occludins $[4,5]$. Hyperglycaemic conditions can also contribute to thickening of the basement membrane, which negatively impacts on the survival of the endothelium and can increase vascular permeability [6]. Other features of diabetic retinopathy include the formation of acellular capillaries, resulting from migration or loss of pericytes from the thick basement membrane [6]. Further inflammation occurs during early onset of diabetic retinopathy [7]. This inflammation includes activation of the endothelium and microglia, as observed in the inner retina in animal models and human post-mortem tissues [7, 8].

Loss of capillaries within the retina, as occurs with diabetic retinopathy, decreases blood perfusion and creates ischaemic/ hypoxic conditions. The sustained hyperglycaemia and hypoxia leads to the induction of vascular endothelial growth factor (VEGF), a powerful stimulator of both neovascularisation and vascular leakage in people with diabetes [9]. VEGF/VEGF receptor 2 (VEGFR2) signalling results in phosphorylation and internalisation of VE-cadherin, inducing vascular permeability $[10,11]$. A number of retinal cells, such as Müller cells, neurons and pigment epithelium cells, are sources of VEGF, making diabetic retinopathy a disease involving multiple cell types [12].

Current treatments for PDR and DMO include laser photocoagulation and vitrectomy surgery. In addition, anti-VEGF treatments are being used as supplementary or stand-alone options, with steroids and other anti-inflammatory agents reserved for poor responders to anti-VEGF therapies [13]. However, poor response and the risk of traction retinal detachment seen in PDR with certain anti-VEGF agents means there is an urgent need for alternatives in drug treatment [13].

VE-cadherin is critical in maintaining the integrity of the endothelial cell barrier [11] and attenuates VEGF signalling, thus limiting angiogenesis [14]. A marked reduction in levels of VE-cadherin is seen in human retina obtained post-mortem from individuals with diabetes [15], while increased plasma levels have been observed in individuals with type 2 diabetes, suggesting that it is shed from the endothelium [16]. Higher levels of phosphorylation and degradation of VE-cadherin have been observed with increased vascular leakage in diabetic rodents $[5,17,18]$. Thus, a drug that restores the levels of VE-cadherin may have profound effects on the pathology of diabetic retinopathy, limiting vascular leakage and inhibiting angiogenesis.

We have previously reported the development of a novel oligonucleotide-based technology (termed Blockmirs) that regulates microRNA (miRNA)-mRNA interactions by binding to the miRNA-binding site in the specific target mRNA at the 3 '-untranslated region and inhibiting the miRNA target gene. The Blockmir CD5-2 prevents the miR-27a-mediated decrease in transcription of VE-cadherin, resulting in an increase in expression of the endogenous protein in endothelial cells. CD5-2 improves vascular integrity, inhibits angiogenesis and also enhances blood flow recovery in a mouse model 

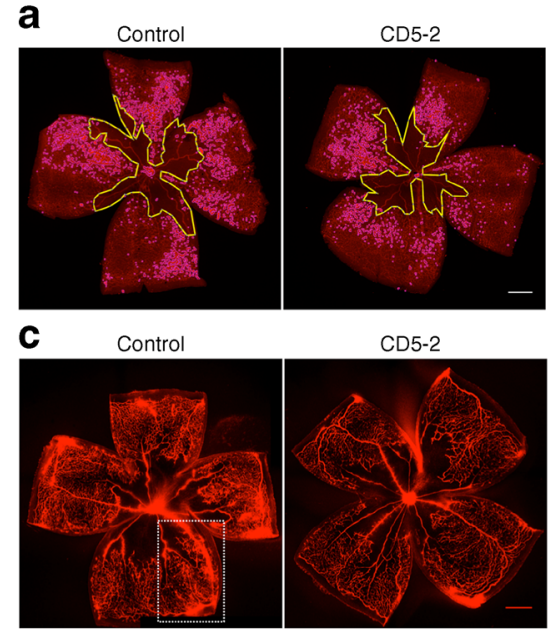

Fig. 2 CD5-2 improves vascular leakage in retinas. (a) OIR retinas at P17 stained with isolectin-B4 for neovascularisation. Scale bar, $500 \mu \mathrm{m}$. (b) Quantification of neovascularisation (magenta in a) and avascular area (yellow outline in a) between control and CD5-2-treated mice. (c) OIR retinas at $\mathrm{P} 17$ perfused with Evans Blue dye (red). The rectangle shows an

of hindlimb ischaemia [19]. Furthermore, CD5-2 normalises the vasculature in tumour-associated vessels, enhancing the effect of immunotherapy [20]. As high levels of miR-27 have been found in the blood of people with diabetic retinopathy and in mouse choroidal neovascularisation samples [21-23], we investigated whether CD5-2 promotes VE-cadherin expression in diabetic retinopathy.

\section{Methods}

Animals All animal studies were approved by Royal Prince Alfred Hospital Animal Welfare Committee or the Sydney University Animal Ethics Committee, Sydney, NSW, Australia. C57BL/6J mice purchased from the Animal Resources Centre (Canning Vale, WA, Australia) and Australian BioResources (Moss Vale, NSW, Australia) were used for the oxygeninduced retinopathy (OIR) model and streptozotocin (STZ)induced diabetes model, respectively. Blockmir CD5-2 and controls (LGC Axolabs, Kulmbach, Germany) were injected at $30 \mathrm{mg} / \mathrm{kg}$ and analysed blind in all experiments except the Müller cell-knockout (KO) model, where only mice in this model showing a similar extent of focal lesions were given the drug. All mice were fed standard laboratory chow, received water ad libitum and were housed in a temperature-controlled environment with a $12 \mathrm{~h} \mathrm{light/dark} \mathrm{cycle} \mathrm{(lights} \mathrm{on} \mathrm{at} \mathrm{06:00} \mathrm{hours} \mathrm{and}$ off at 18:00 hours every day).

Blockmir sequence The underlined oligonucleotides are locked nucleic acid monomers and the remainder consist of 2'-O-methyl-RNA monomers.
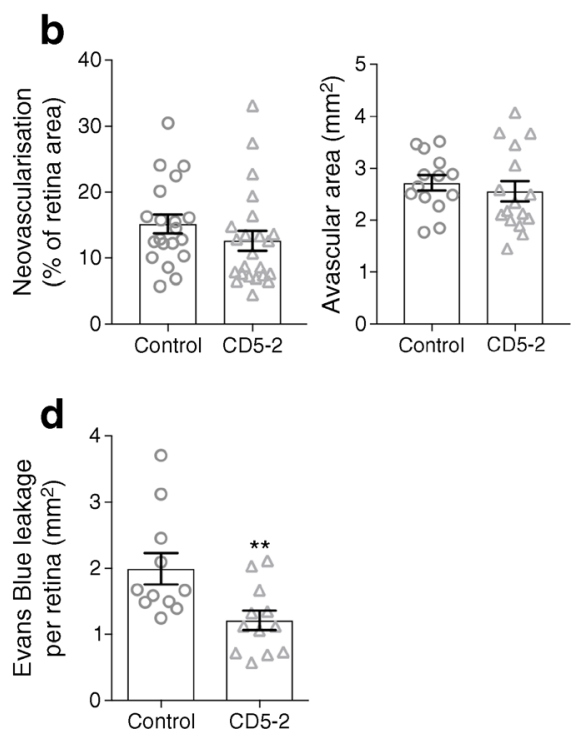

example area of leakage. Scale bar, $500 \mu \mathrm{m}$. (d) Quantification of leakage area per retina. Data in (b) and (d) are the mean \pm SEM of three independent experiments, with representative images shown in (a) and (c); control-treated mice, $n=7$; CD5-2-treated mice, $n=8$; ${ }^{*} p<0.01$ vs controltreated mice by unpaired Student's $t$ test

\section{CD5-2: 5'-ITmUmCmAmCmAmGmUITIGICITITICIA-3'}

Control: 5'- ITmCICmAIGmAIGmAITmGmGITmUIGIA-3'

Generation of conditional Müller cell-KO model This study was performed in accordance with the Association for Research in Vision and Ophthalmology statement. The Müller cell-KO model was generated by crossing tamoxifendependent recombinase Müller cell-CreER (Tg(Rlbp1-Cre/ ERT2)1Wshn; CBA background) mice with Rosa-DTA176 mice (129-Gt(ROSA)26Sor ${ }^{\text {tm1(DTA)Mrc }}$ C57BL/6 J background). Müller cell ablation was induced by intraperitoneal injection of tamoxifen at 8-10 weeks of age for 4 days, as described previously [24]. After 3 months, mice with established focal vascular lesions were injected intravenously with a single dose of CD5-2 or control. Vascular lesions were monitored using fluorescein angiography for 21 days. See electronic supplementary material (ESM) Methods for more details.

Fundus fluorescein angiography Fundus fluorescein angiography was performed on Müller cell-KO transgenic mice ( $n=5$ controls and $n=6$ CD5-2-treated mice) using a modified clinical fundus camera (Topcon TRC-50VT, Oakland, NJ, USA) mounted with a 40D lens as previously described [25].

Mice with streptozotocin-induced diabetes Diabetes was induced in male mice, 10 weeks old, with an intraperitoneal injection of $180 \mathrm{mg} / \mathrm{kg}$ streptozotocin (Sigma, St Louis, MO, USA). Control mice were injected intraperitoneally with $0.1 \mathrm{mmol} / 1$ citrate buffer. CD5-2 or control was given 
Fig. 3 VE-cadherin expression is increased following CD5-2treatment. (a-f) Comparison of VE-cadherin expression in arteries, veins and capillaries of superficial plexus between control and CD5-2-treated diabetic mice. Data are the mean \pm SEM of two independent experiments, with representative images; control-treated diabetic mice, $n=9$; CD5-2-treated diabetic mice, $n=8 ; * p<0.05$, $* * p<0.01$ vs diabetic control VE-cadherin mean fluorescence intensities, by unpaired Student's $t$ test. Scale bars, $50 \mu \mathrm{m}(\mathbf{a}, \mathbf{b})$ $25 \mu \mathrm{m}(\mathbf{c})$. (g, h) VE-cadherin expression in capillaries of the retinal deep plexus was assessed in diabetic mice treated with control and CD5-2. The white square areas are displayed as magnified images below each image. Data are the mean \pm SEM of three independent experiments, with representative images; nondiabetic mice, $n=4$; controltreated diabetic mice, $n=11$; CD5-2-treated diabetic mice, $n=$ $10 ; * * p<0.01$ vs control-treated diabetic mice by Dunnett's multiple comparisons test. Scale bars, $25 \mu \mathrm{m}$. A, artery; C, capillary; V, vein; VEC, VEcadherin
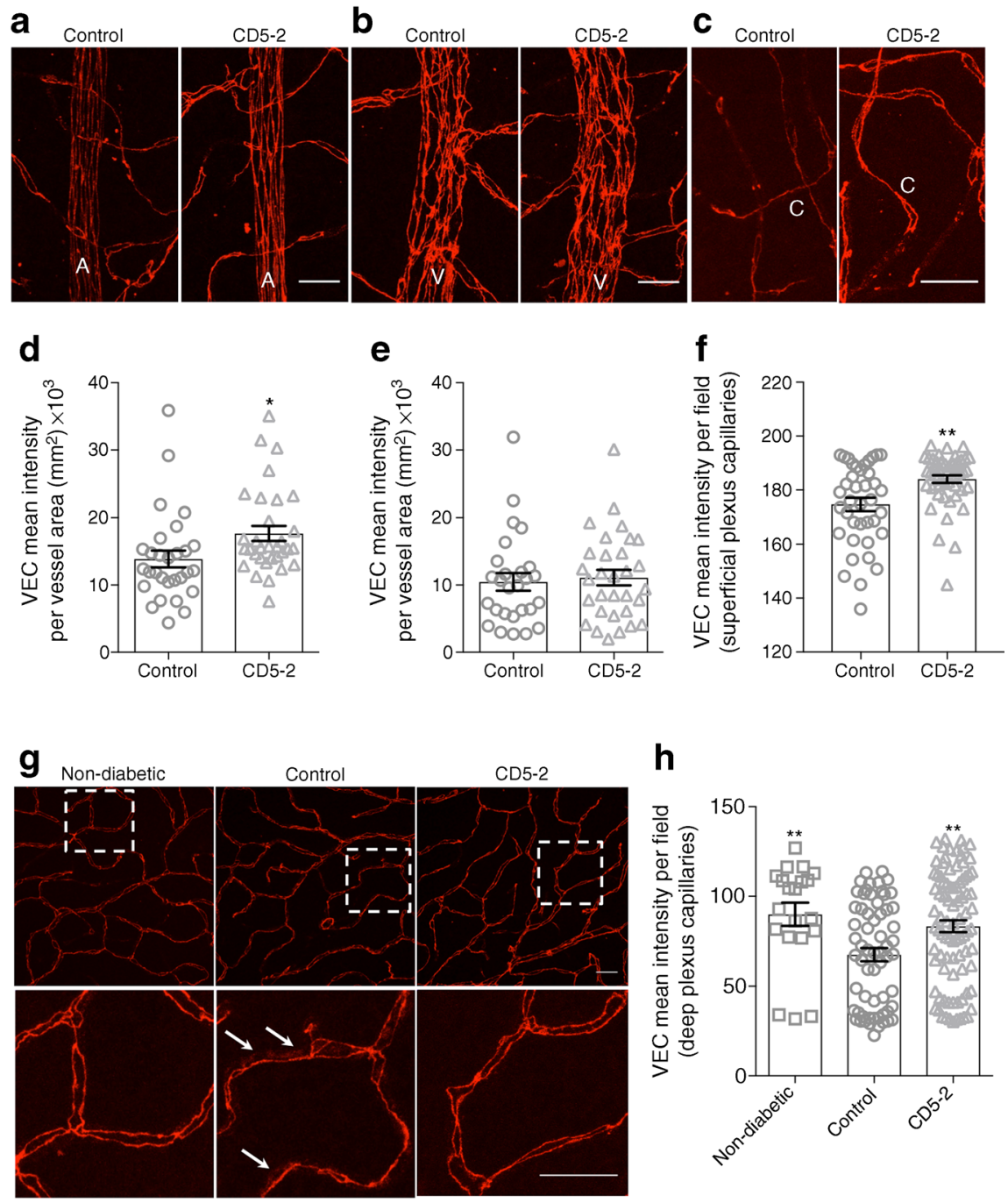

intravenously to diabetic mice on day 11 . On day 14 , retinas were collected to assess for vascular permeability and stained for retinopathy changes. See ESM Methods for more details.

Oxygen-induced retinopathy OIR was induced by exposing pup litters to $75 \%$ oxygen from 7 days postnatal (P7) to 12 days postnatal (P12). Pups were returned to room air from P12 to 17 days postnatal (P17). CD5-2 or control was given intraperitoneally to mice at P12 and 14 days postnatal (P14). Mice were euthanised at P17 and retinas were collected. See ESM Methods for more details.

Retinal flat-mount immunostaining The immunostaining method for whole-mount retina was adapted from Shen et al [24]. Retinas collected from STZ diabetic mice and OIR mice were stained for early and proliferative retinopathy features, respectively. Antibodies for neural/glial antigen 2 (NG2), VEcadherin, claudin-5 and CD45 were used to assess pericyte coverage, endothelial junction integrity and microglia density and morphology, respectively. See ESM Methods for more details.
Evans Blue leakage assay Evans Blue was injected into mice to assess vascular leakage in retinas. See ESM Methods for more details.

In situ hybridisation Fluorescence in situ hybridisation using a 20-mer antisense oligonucleotide probe conjugated to 6carboxyfluorescein (FAM) (Ribotask ApS, Odense, Denmark) was used to detect CD5-2 localization in the retinal vasculature. This method was adapted from Zhao et al [20]. CD31 was used as a general marker for vasculature. See ESM Methods for more details.

Cell culture conditions HUVECs were isolated from donated cords with approval from the Royal Prince Alfred Hospital Human Ethics Committee (X16-0225). The VE-cadherin null (VEC-null) endothelial cell line was kindly given by E. Dejana (Milan, Italy) and the mouse brain endothelial cell line was provided by G. Grau (Sydney, Australia). These cells were grown in medium 199, EBM2 (endothelial cell growth basal medium) and DMEM, respectively, with supplements at 


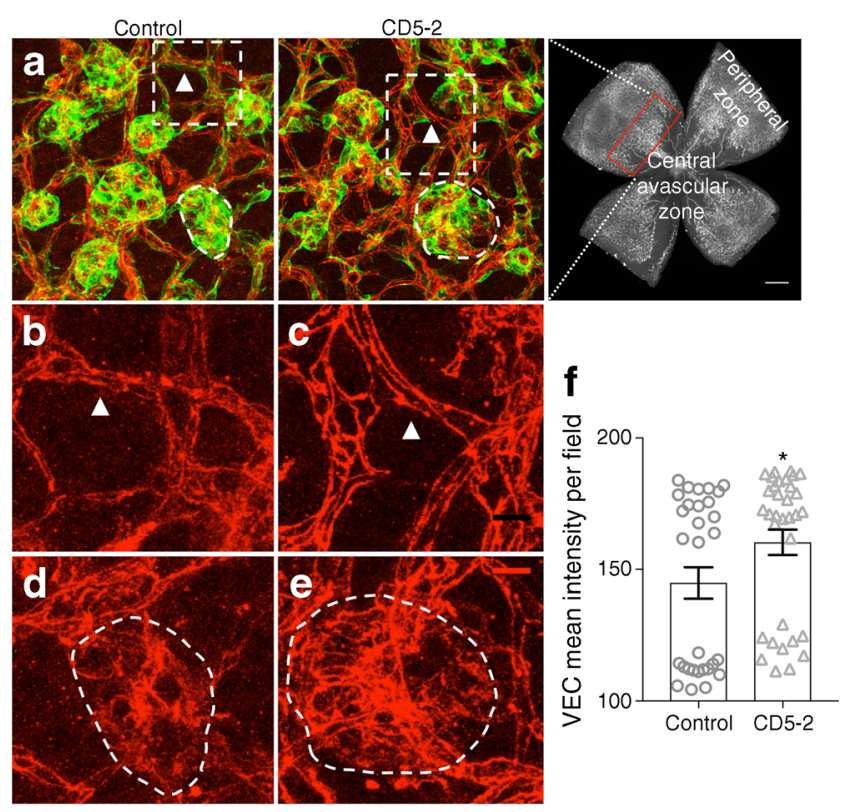

g
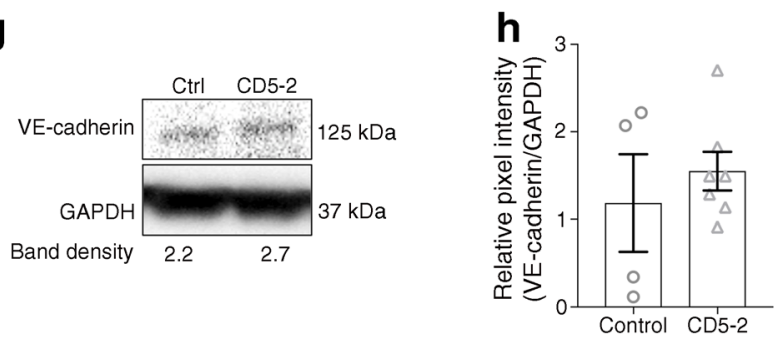

Fig. 4 VE-cadherin is protected in neovascular tufts and surrounding microvessels. (a) Comparison of VE-cadherin (red) in microvessels and neovascular tufts between control and CD5-2-treated mice in areas adjacent to the central avascular area, as outlined by red box. Neovascular tufts were identified morphologically using an anti-NG2 antibody (green) staining. (b-e) Magnifications of areas shown with dashed borders in (a); the circular areas and white arrowheads highlight areas of VE-cadherin preservation by CD5-2 in microvessels and tufts, respectively. Scale bars: $25 \mu \mathrm{m}(\mathbf{a}), 10 \mu \mathrm{m}(\mathbf{b}-\mathbf{e})$. (f) VE-cadherin expression in the microvessels and tufts was measured as mean intensity. Data are the mean $\pm \mathrm{SEM}$ of three independent experiments, with representative images shown in (ae); control-treated mice, $n=7$; CD5-2-treated mice, $n=8$; $* p<0.05$ vs control-treated OIR at P17, by unpaired Student's $t$ test. (g) Western blot analysis for the expression of retinal VE-cadherin in OIR P17 mice treated with control or CD5-2. Glyceraldehyde-3-phosphate dehydrogenase (GAPDH) was used as loading control. (h) Change in VE-cadherin level was measured as pixel density and normalised to GAPDH. Data are the mean $\pm \mathrm{SEM}$ of two independent experiments, with representative image shown in (g); control-treated mice, $n=4$; CD5-2-treated mice, $n=6$. Ctrl, control; VEC, VE-cadherin

$37^{\circ} \mathrm{C}$ and $5 \% \mathrm{CO}_{2}$. Cells were cultured in six-well plates and transfected with CD5-2 or control Blockmir at $15 \mathrm{nmol} / \mathrm{l}$ for $48 \mathrm{~h}$ using HiPerfect transfection reagent (Qiagen, Hilden, Germany) in Opti-MEM reduced serum media (Gibco, Waltham, MA, USA). See ESM Methods for details.

Western blot Lysates were collected to determine effects on TGF- $\beta$ and wingless/integrated (WNT) signalling pathways and levels of VE-cadherin, Smad family member (SMAD) 2/3 activity, LDL receptor-related protein 6 (LRP6) and plateletderived growth factor, B polypeptide (PDGF-B) expression. Retinal homogenates were collected from OIR mice to test for VE-cadherin and claudin-5. See ESM Methods for more details.

Image analysis Retinas were imaged using Leica SP5 confocal microscope (Leica Microsystems, Wetzlar, Germany). ImageJ software (version 1.52e) was used to analyse pericyte count and morphology, capillary density, VE-cadherin and claudin-5 expression, microglia density and morphology. See ESM Methods for more details.

Statistical analysis In experiments in which a single experimental group was compared with a single control group, statistical comparisons were made by unpaired Student's $t$ test. In experiments in which multiple experimental groups were compared with a control group or in which there were comparisons among multiple experimental groups, the Kruskal-Wallis nonparametric test was performed. A two-way ANOVA was used to assess Sholl curves of microglia morphology, followed by Šidák's multiple comparisons test. All comparisons were twotailed unless otherwise indicated. A $p$ value of less than 0.05 was considered significant. In figures, data displayed with error bars represent the mean $\pm \mathrm{SEM}$.

\section{Results}

CD5-2 reduces vascular leakage in tamoxifen-inducible Müller cell-KO mice We used the Müller cell-KO model to determine whether CD5-2 has effects in the eye, as previous studies have shown that loss of these cells induces retinal vascular leakage [24]. Müller cell depletion is induced by tamoxifen and intraretinal neovascularisation generally develops over 3 months. Vascular leakage is visualised by fundus fluorescein angiography, with lesions ranging from mild to severe (Fig. 1a). Mice that developed vascular leakage of medium to severe grades were selected and CD5-2 or control oligonucleotide delivered intravenously. Changes in vascular leakage were periodically monitored non-invasively by angiography for 3 weeks. Mice treated with CD5-2 had fewer lesions with leakage and a lower grade of vascular leakage than those treated with control, even to 21 days post-injection (Fig. 1b, c). We noted that, with CD5-2 administration, the vascular leakage in some lesions totally disappeared or diminished in size (arrow), whereas most lesions in mice treated with the control showed enlarged areas of vascular leakage with time (arrow head).

CD5-2 inhibits retinal vascular leakage in models of experimental diabetic retinopathy The STZ-induced diabetes model can develop a retinopathy similar to that in humans, with features that include loss of pericytes, pre-retinal neovascularisation and vascular leakage. Hyperglycaemia, as defined by 
Fig. 5 In situ hybridisation detection of CD5-2 in the OIR model. (a) Blood vessels in retina cryosections were stained using an anti-CD31 antibody (red). CD5-2 (green) was detected by in situ hybridisation using a 6carboxyfluorescein (FAM) probe, $6 \mathrm{~h}$ after administration to $\mathrm{P} 14$ mice. Dashed ovals show areas of CD5-2 colocalisation in CD31positive blood vessels. The GCL, INL and ONL layers in the retina were distinguished using DAPI nuclear labelling. (b) Specificity of detection using no-probe as control. Yellow arrows show the lack of CD5-2 staining in the blood vessels. Representative images from CD5-2-treated mice are shown, $n=4$. Scale bar, $50 \mu \mathrm{m}$
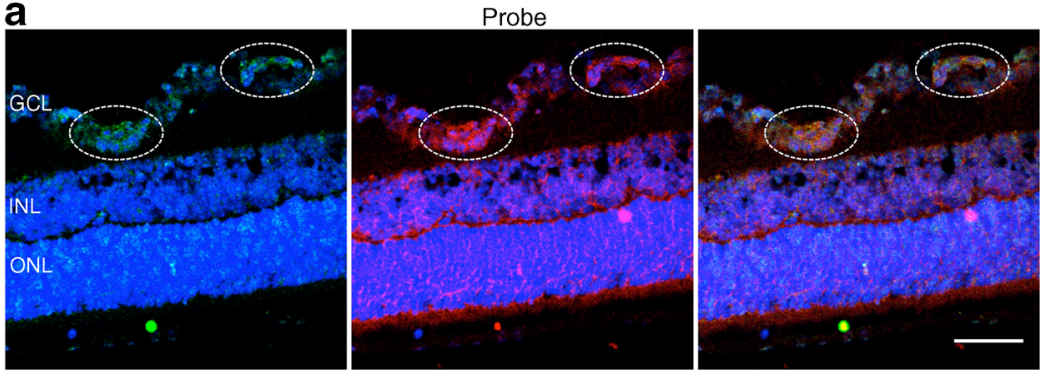

b

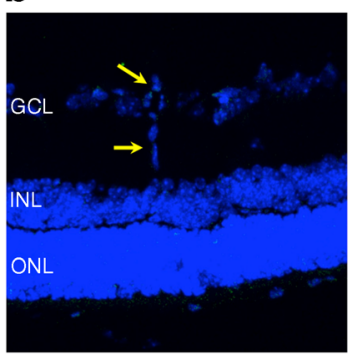

No-probe control

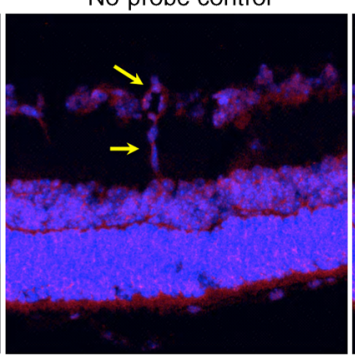

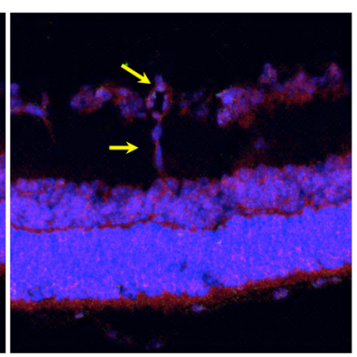

glucose $>20 \mathrm{mmol}$ in the blood, was induced 3 days after a single dose of STZ in approximately $40 \%$ of mice (ESM Table 1). Hyperglycaemia was sustained for up to 14 days, the endpoint of the experiment. On day 11, mice showing similar levels of hyperglycaemia were injected with either CD5-2 or control and analysed 3 days later. CD5-2 and control had no significant effect on hyperglycaemia when compared with diabetic mice (ESM Fig. 1a). Further, they did not affect glucose levels in normal mice (ESM Fig. 1b). Vascular leakage in the STZ-induced diabetes model was assessed using Evans Blue dye perfusion. At 2 weeks after STZ-induced diabetes, some mice developed increased vascular leakage in the retina compared with non-diabetic mice (ESM Fig. 2a). The vascular leakage was observed in areas of intermediatesized arterioles, though the amount of leakage and number of mice developing this leakage were variable; overall, the vascular leakage induced in the diabetic animals was mild. There was, however, a trend for CD5-2-treated and non-diabetic mice to show less vascular leakage, but the changes did not reach statistical significance (ESM Fig. 2b). Therefore, we turned to the OIR model, a proliferative model of diabetic retinopathy that shows gross features of vascular leakage [24].

The OIR model is characterised by significant hypersprouting, neovascular tuft formation that protrudes into the vitreous [25] and increased vascular permeability, typically peaking at P17. CD5-2 was administered intraperitoneally at P12 and P14, and mice were examined at P17. There was no significant difference in avascular areas and neovascularisation tuft size between the control and CD5-2 groups (Fig. 2a, b). However, when vascular leakage was assessed, control-treated mice displayed significant vascular leakage (rectangle in Fig. 2c), whereas the dye leakage was significantly reduced in CD5-2-treated mice (Fig. 2c, d).
CD5-2 increases VE-cadherin expression Analysis of VEcadherin levels showed that arteries, arterioles and capillaries within the superficial layer of the retina in mice given CD5-2 had higher levels of VE-cadherin compared with controltreated mice (Fig. 3a, c, d, f). There was no change in the levels of VE-cadherin in retinal veins between the two groups (Fig. 3b, e). In diabetic mice receiving control, VE-cadherin was found to be discontinuous or diffuse at the junctions of deep retinal capillaries (Fig. 3g) compared with non-diabetic animals, consistent with previous reports [5, 18]. In contrast, CD5-2-treated diabetic mice displayed continuous VEcadherin expression at the junctions of microvessels in the deep plexus, with the level of VE-cadherin expression similar to that seen in non-diabetic animals (Fig. 3g, h).

We next analysed vessel morphology in the OIR model, particularly in regions located at the border of the central avascular area and adjacent vascular areas. Pathological neovascularisation was highest in these areas in the OIR retina at P17 [26]. Neovascular tufts were outlined by NG2 staining for pericytes (green) (Fig. 4a). White arrowheads showed that VE-cadherin expression in the microvessels proximal to the neovascular tufts was diffuse and discontinuous in controltreated mice (Fig. 4b). However, VE-cadherin expression was increased in neovascular tufts in CD5-2-treated mice (Fig. 4c, e). Collectively, VE-cadherin expression in the microvessels and tufts was significantly higher in CD5-2treated mice compared with mice receiving control (Fig. 4f). Consistent with this, CD5-2 increased VE-cadherin levels approximately twofold compared with controls in retinal lysates analysed by western blots (Fig. 4g, h). Surprisingly, when we assessed vessels in the peripheral vasculature, there was no difference in levels or in localisation of VE-cadherin between control and CD5-2-treated animals (ESM Fig. 3), suggesting 

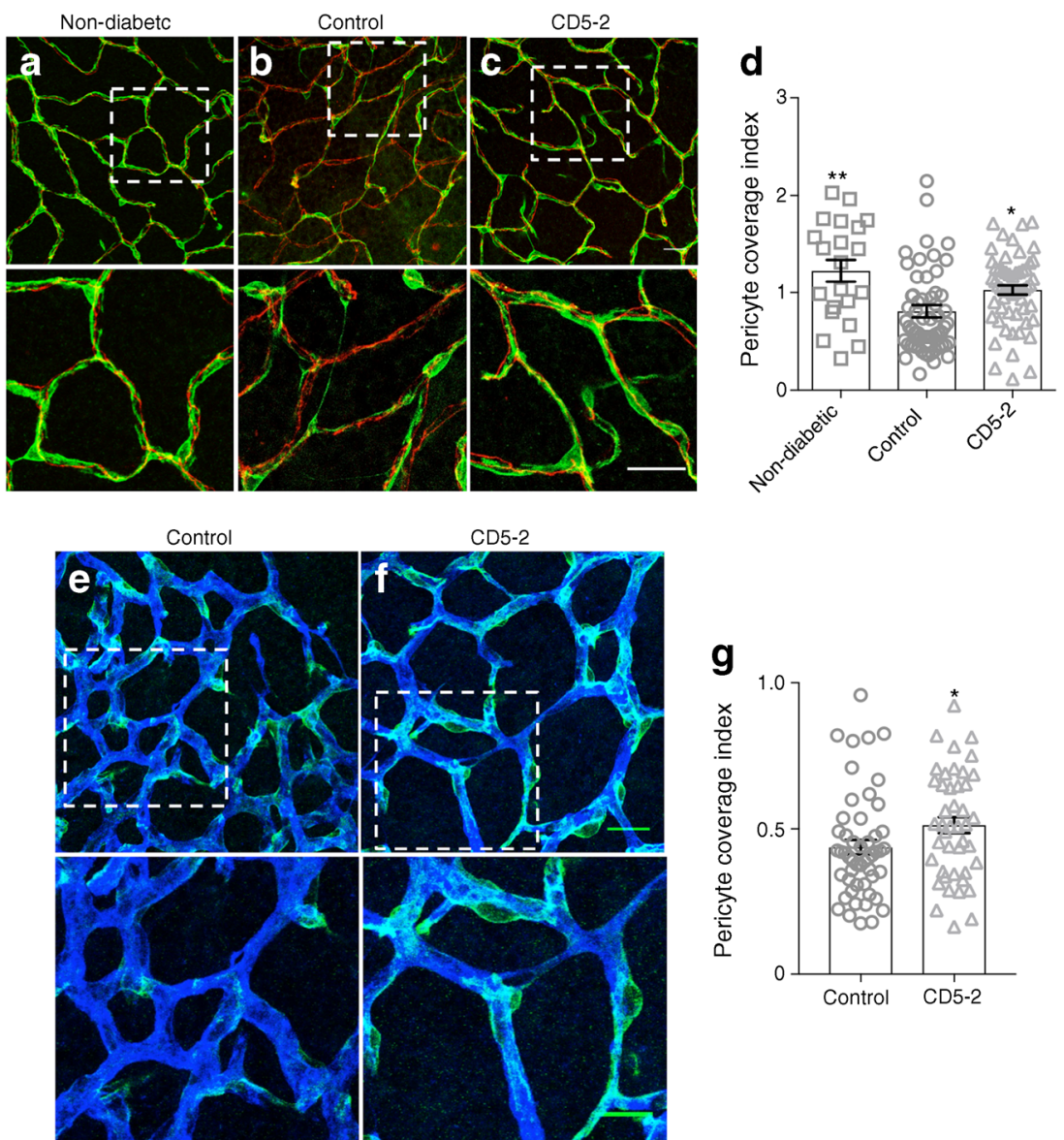

Fig. 6 CD5-2 maintains pericyte coverage. (a-c) Pericyte coverage on VE-cadherin-positive capillaries (red) in the deep plexus using anti-NG2 antibody (green). The white square areas are displayed as magnified images underneath each representative image. Scale bar, $25 \mu \mathrm{m}$. (d) Pericyte coverage index was expressed as NG2-positive area relative to VE-cadherin-positive capillary area. Data are the mean \pm SEM of three independent experiments, with representative images shown in $(\mathbf{a}-\mathbf{c})$. Non-diabetic mice, $n=4$; control-treated diabetic mice, $n=11$; CD5-2treated diabetic mice, $n=10 ; * p<0.05$, ** $p<0.01$ vs diabetic control by Kruskal-Wallis non-parametric test for multiple comparisons between

that VE-cadherin expression in this region is less affected by hyperoxia vaso-obliteration and hypoxia-driven neovascularisation. Claudin-5 distribution in areas of neovascular tufts and in arterioles and whole-retinal protein expression revealed a slight increase in localisation to endothelial junctions in CD5-2-treated mice (ESM Fig. 4a), though there was no increase in claudin-5 levels as quantified from overall intensity (ESM Fig. 4b) or by western blots (ESM Fig. 4c, d).

CD5-2 expression is detected in the vasculature Given the effects of CD5-2 on the vasculature, we confirmed that CD5-2 is detected in the endothelium. Using in situ hybridisation of retinal cryosections of OIR at P14, CD5-2 was strongly localised to the vasculature in the ganglion cell layer (GCL), $6 \mathrm{~h}$ post-injection of the drug (Fig. 5a, dashed ovals). Yellow arrows show the lack of staining in vessels in groups. (e, f) Pericyte coverage in control or CD5-2-treated OIR P17 mice: NG2 staining (green) for pericytes and isolectin-B4 (blue) for deep retinal vessels. White square areas are displayed as magnified images underneath each image. Scale bar, $25 \mu \mathrm{m}$. (g) Pericyte coverage index was expressed as NG2-positive area relative to isolectin-B4 positive vessels. Data are the mean \pm SEM of three independent experiments, with representative images shown in (e, f). Control-treated mice, $n=7$; CD5-2treated mice, $n=8 ; * p<0.05$ vs control-treated mice by unpaired Student's $t$ test

the no-probe control, demonstrating the specificity for CD5-2 (Fig. 5b). The non-specific CD31 staining in the inner nuclear layer (INL) and outer nuclear layer (ONL) is likely due to the use of the mouse monoclonal antibody. Although there is some CD5-2 staining in the ONL and INL, this is probably caused by diffusion into the retinal tissue.

CD5-2 maintains pericyte coverage Pericyte dropout is an early hallmark of diabetic retinopathy [27-29]. Microvessels in non-diabetic mice had significant pericyte coverage (Fig. 6a, d). In CD5-2-treated diabetic mice, pericyte coverage on the microvessels was significantly retained compared with the control-treated diabetic mice, with levels approaching those seen in non-diabetic microvessels (Fig. 6b-d). In the OIR model, pericytes in control-treated mice appeared severely contracted as shown by the flattened cell bodies and lack of 

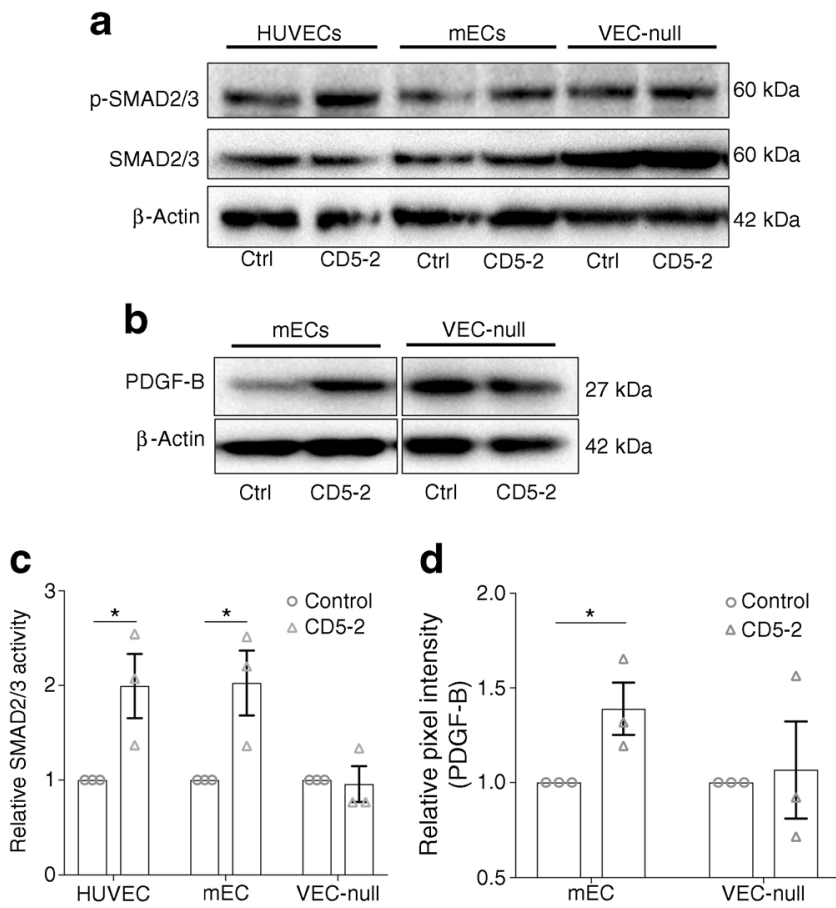

Fig. 7 CD5-2 results in activation of TGF- $\beta$ and PDGF-B signalling pathways in the endothelium. (a) Phosphorylation of SMAD2/3 and total SMAD2/3 in lysates of HUVECs, mouse brain endothelial cells and VEC-null mouse endothelial cells $24 \mathrm{~h}$ after control and CD5-2 transfection. $\beta$-Actin was used as loading control. (b) The expression of PDGF-B in mouse brain endothelial cells and VEC-null mouse endothelial cell lysates $24 \mathrm{~h}$ after control and CD5-2 transfection. $\beta$-Actin was used as loading control. (c) Quantification of densitometric ratio for SMAD2/3 activity. (d) PDGF-B expression was measured as pixel density and normalised to $\beta$-actin. All data are presented as the normalised expression of mean \pm SEM of three independent experiments. $* p<0.05$ vs controltreated cells by paired Student's $t$ test. Circles, control; triangles, CD52. Ctrl, control; mEC, mouse brain endothelial cell; VEC-null, VEcadherin-null mouse endothelial cell

processes along the vasculature (Fig. 6e). In contrast, the pericyte coverage index in CD5-2 treated mice was significantly greater in the remaining deep plexus microvessels, as evidenced by pericyte processes extending along the vasculature (Fig. 6f, g).

CD5- 2 regulates the TGF- $\beta$ and PDGF-B pathways through VEcadherin TGF- $\beta$ and WNT signalling pathways have been implicated in diabetic retinopathy $[30,31]$. To determine whether CD5-2 alters these pathways, HUVECs, mouse brain endothelial cells and VEC-null mouse endothelial cells were treated with control or CD5-2. We determined the expression of $p$-SMAD2 $/ 3$ as a measure of the TGF- $\beta$ pathway and $p-$ LRP6 for the WNT pathway. PDGF-B, which promotes pericyte recruitment through the PDGF receptor (PDGFR)- $\beta$ /PDGF-B axis [32] was also determined. CD5-2 treatment upregulated VE-cadherin in HUVECs and mouse brain endothelial cells but not in VEC-null mouse endothelial cells (ESM Fig. 5a). CD5-2 increased expression of pSMAD2/3 and PDGF-B in both HUVECs and mouse brain endothelial cells (Fig. 7a-d), but the changes were dependent on VE-cadherin, as no increases were seen in the VEC-null cells (Fig. 7a-d). There was no consistent change in the levels of p-LRP6, suggesting CD5-2 has no major effect on the WNT signalling pathway (data not shown).

Microglia activation is reduced by CD5-2 in the deep plexus Microglia are known to be activated in diabetic retinopathy and contribute to ongoing inflammation. Microglia in the inner retina were stained for the immune-cell marker, CD45. However, it was difficult to distinguish between perivascular macrophage and microglia in the superficial plexus. Therefore, we analysed microglia in the deep plexus, where their morphology is more easily defined. Highly ramified microglia (as judged by the small size of the cell body and the length and number of processes) can be observed evenly distributed in the non-diabetic retina (Fig. 8a, d). In control-treated diabetic animals, the microglia had fewer branches and processes, indicative of activation (Fig. 8b, e). CD5-2-treated animals showed morphology similar to that seen in non-diabetic animals, with a high degree of branching and processes (Fig. 8c, f). Manual quantification of microglia numbers showed also that there was a significant reduction in the number of microglia in the control-treated retina when compared with CD5-2-treated and non-diabetic retina (Fig. 8g). The length of processes, as measured by the distance from the cell soma, and number of branches showed that microglia from CD5-2-treated animals displayed a less-activated morphology compared with control-treated diabetic animals (Fig. 8h).

\section{Discussion}

Diabetic retinopathy is associated with loss of vascular-related cells, including Müller cells, pericytes and endothelial cells. Their loss, which has been attributed to oxidative stress and inflammation, leads to BRB breakdown, evidenced by oedema, neovascularisation, retinal dysfunction and, ultimately, blindness. In this study, we demonstrate that our recently developed drug, CD5-2, penetrates the vasculature of the eye, significantly inhibits vascular leakage and promotes pericyte coverage, thus acting to preserve the BRB. Consistent with the known target of CD5-2, there was an increase in levels of VEcadherin following CD5-2 treatment in the vessels where leakage is a feature. In addition, CD5-2 restored the microglia population found in the deep plexus towards a less-activated state, similar to that seen in normal retina.

CD5-2 significantly increases the distribution of VE-cadherin at the endothelial junctions in microvessels in the diabetic retinopathy model and induces an increase in the overall protein expression of VE-cadherin in the OIR model. This is consistent with our previous reports showing VE-cadherin to be a target for CD5-2 $[19,20]$. The increase in vascular leakage in both 
Fig. 8 Microglia density and morphology in the diabetic retina is similar to non-diabetic retina after CD5-2 treatment.

Representative images of retinal microglia numbers $(\mathbf{a}-\mathbf{c})$ and morphology $(\mathbf{d}-\mathbf{f})$ in the deep plexus of non-diabetic mice and control- and CD5-2-treated diabetic mice. Microglia were labelled using anti-CD45 antibody (red). Scale bar, $50 \mu \mathrm{m}$ (a-c); and $25 \mu \mathrm{m}(\mathbf{d}-\mathbf{f})$. (g) Microglial density was measured as number of microglia in the deep plexus per field. Data are the mean \pm SEM of three independent experiments. Non-diabetic mice, $n=5$; control-treated diabetic mice, $n=9$; CD5-2-treated diabetic mice, $n=8 ; * * * p<0.001$ vs diabetic control by unpaired Student's $t$ test. (h) Sholl analysis of the branches and processes of microglia. Data are mean \pm SEM of two experiments. Non-diabetic, $n=60$ cells; control, $n=90$ cells; CD5-2, $n=100$ cells; $* * p<0.01$ and $* * * p<0.001$ vs controltreated diabetic mice, by Šidák's multiple comparisons test. Db, diabetic
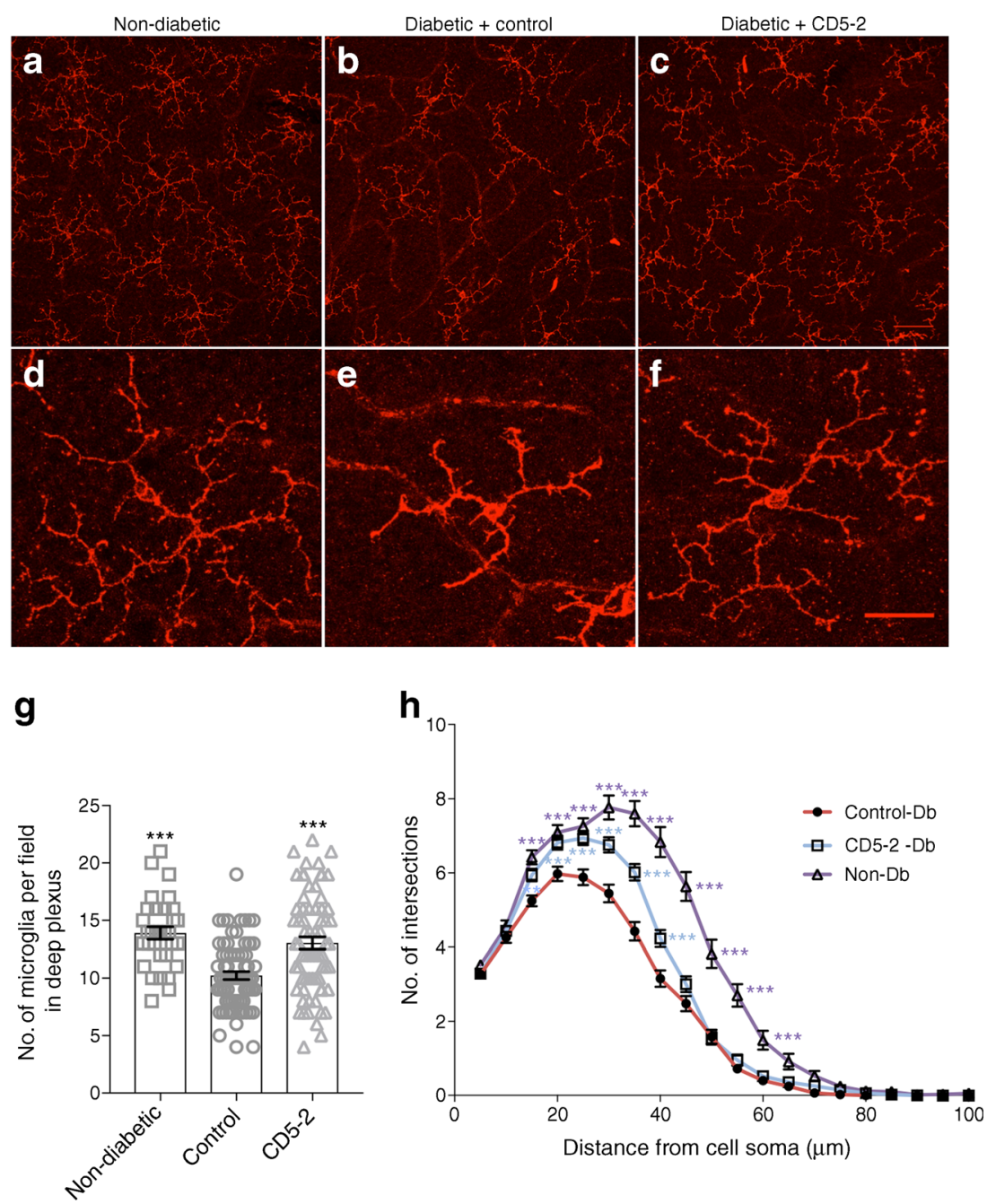

experimental models is associated with a decrease in VEcadherin, similar to that reported previously [5], though in the OIR model there is loss of VE-cadherin from neovascular tufts and microvessels adjacent to the tufts, but not from the peripheral vasculature. There appear to be site-specific differences in VEcadherin based on kinetics, computational modelling and vascular dynamics in the neovascular tufts and vessels in the peripheral zone, which may account for the site-specific effects of CD5-2 $[26,33]$. Although claudin-5 expression can be regulated through VE-cadherin [34], there were no significant changes with CD5-2. Similar to a previous report [35], we found no significant loss or reorganisation of claudin-5 in the OIR model (ESM Fig. 4), suggesting longer-term models of diabetic retinopathy maybe required to elucidate changes in claudin-5.

The second major effect induced by CD5-2 is the preservation of pericyte coverage. In the central nervous system, pericytes play essential roles in angiogenesis and vessel stabilisation, via the PDGFR- $\beta$ /PDGF-B axis [32], and in maintenance of vessel barrier, immune-cell trafficking and regulation of blood flow [36]. Pericyte loss is an initial event in the development of diabetic retinopathy [1]. Co-culture studies have shown that pericytes can increase levels of VE-cadherin in an endothelial cell monolayer and decrease vascular permeability [37]. Thus, pericyte dropout could be either the cause of junctional dysfunction or a consequence. The preservation of pericyte numbers by CD5-2 treatment was substantiated by our in vitro studies, in which CD5-2 increased protein levels of PDGF-B in endothelial cells. Further, PDGF-B is a downstream product of the TGF- $\beta$ pathway [38], and CD5-2 also increased SMAD2/3 activity, in line with a previous study showing VE-cadherin to be a regulator of TGF- $\beta$ signalling [39]. Importantly, VEC-null mouse endothelial cells showed no changes in PDGF-B or SMAD2/3 expression, demonstrating the VE-cadherin dependence of these effects. Previously, we have shown that CD5-2 can induce activation of endothelial nitric oxide synthase (eNOS) [20]. Diabetic eNOS-KO mice develop accelerated features of retinopathy associated with pericyte loss [40], suggesting CD5-2 may also improve pericyte coverage through eNOS regulation.

CD5-2 treatment inhibited vascular leakage in conditional Müller cell-KO mice. Müller cells are the only glia cells that can span the entire width of the retina and have interactions with almost every cell type. As such, loss of Müller cells through the 
progression of diabetic retinopathy [41] impacts on the integrity of the BRB [24]. Our results show that a single dose of CD5-2 can significantly reduce focal vascular leakage for at least 21 days post-treatment. The 2'-O-methyl modifications to the RNA monomers in CD5-2 allow for increased hybridisation affinity and resistance to base hydrolysis and nuclease activity, and for extended residence time of the drug in the tissue [42], suggesting a mechanism for its long duration of activity. The effect of CD5-2 was specific to the vascular leakage component of dysfunctional vessels, as it did not significantly reduce neovascularisation or avascular regions in the OIR retinas. This is in line with our previous report of CD5-2 in tumour angiogenesis, in which CD5-2 did not induce vessel pruning [20]; the lack of pruning is likely to limit the hypoxic microenvironment.

Finally, and somewhat surprisingly, CD5-2 had a significant impact on microglial phenotype. Microglia are tissue-resident macrophages, and they are seen clustered around the blood vessels. The progression of diabetic retinopathy is associated with activation of microglia [43]. Activated retinal microglia secrete many growth factors and proinflammatory cytokines, including VEGF, IL- $1 \beta$ and TNF- $\alpha$ [44]. As diabetic retinopathy is classified as a low-grade inflammatory disease, the effect of CD5-2 to reduce microglia numbers and morphology in the deep plexus of the diabetic retina is highly significant. Sholl morphology analysis showed that CD5-2-treated microglia resembled the microglia found in non-diabetic mice, with highly ramified morphology, increased branches and processes. There are many studies that have correlated microglia activation and blood-brain barrier (BBB) leakage [45], though whether microglia activation or BBB breakdown is the initiating event in disease pathology remains unresolved. The mechanism of CD5-2 to limit microglia activation is not known. However, a stabilised vasculature inhibits the secretion of factors that result in microglia/ macrophage activation, such as fibrinogen [46], and activated microglia/macrophages release cathepsin $\mathrm{D}$, a protease that promotes vascular leakage, with subsequent loss of VE-cadherin [47]. Using transcriptomic analysis of the effects of CD5-2 on endothelial cells, we have identified CD5-2-induced changes in the expression of a number of chemokines involved in macrophage activation (data not shown). Further, we have previously demonstrated that CD5-2 exposure results in recruitment of a favourable population of $\mathrm{CD} 8^{+} \mathrm{T}$ cells into the tumour microenvironment [20]. In OIR, the recruitment and expansion of forkhead box P3 (FOXP3) ${ }^{+}$regulatory T cells can reduce vasoobliteration, neovascularisation and vascular leakage, and alter the activation state of microglia [48]. Thus, the mechanism underlying the CD5-2-mediated effects to 'normalise' the vasculature and regulate inflammation in the eye awaits further investigation.

Anti-VEGF therapy has been a breakthrough to counteract pathological angiogenesis and vascular leakage in diabetic retinopathy and DMO. However, anti-VEGF therapy has limitations: not all individuals respond to anti-VEGF therapy [13] and resistance to treatment can develop. Indeed, there are other signalling pathways involved in the pathogenesis of diabetic retinopathy [13]. Finally, the main target of anti-VEGF agents is VEGF-A, which has various isoforms and is known to have heterogeneous activities that play important roles in ocular homeostasis [49]. Thus, there is a need for the development of alternative strategies to target eye disease. Oligonucleotide-based drugs have seen a resurgence in clinical development in the last few years and have effects either as monotherapy or in combination with anti-VEGF therapy [49]. That the unique target of CD5-2, VE-cadherin, has major downstream effects to stabilise barrier integrity and regulate inflammation [34] suggests a clinical utility for CD5-2 in diseases where vascular leakage is central to the pathology.

Acknowledgements We give special thanks to J. Hunter (Centenary Institute, Sydney, Australia) for preparing the human endothelial cells and to the imaging and animal facility staff at the Centenary Institute for their technical assistance. We thank E. Dejana (Italian Foundation for Cancer Research [FIRC], Institute of Molecular Oncology [IFOM], Milan, Italy) for supplying the VEC-null endothelial cells and G. Grau (Department of Pathology, Sydney Medical School, University of Sydney, Sydney, NSW, Australia) for the mouse brain endothelial cells.

Data availability All data generated or analysed during this study are included in this published article and the ESM.

Funding This research was supported by grants from the National Health and Medical Research Council (NHMRC) of Australia (no. 571408). JRG holds the Wenkart Chair of the Endothelium at the Centenary Institute and the Sydney Medical School, University of Sydney.

Duality of interest The authors declare that there is no duality of interest associated with this manuscript.

Contribution statement KKT designed the study, acquired and analysed the data, drafted and approved the final version of the manuscript. JRG and MAV contributed to the conception of the study, and drafted and approved the final version of the manuscript. MG and TM contributed to the conception and design of the study. TC-L was involved in interpretation of the data. WYS, MY, YZ, JL and PC acquired data, revised the article's intellectual content and approved the final version. MG, TM and TC-L revised the article's intellectual content and approved final version. KKT had full access to all the data, excluding those relating to the Müller cell transgenic model in this study. KKT and JRG are responsible for the integrity of this study.

\section{References}

1. Antonetti DA, Klein R, Gardner TW (2012) Diabetic retinopathy. N Engl J Med 366(13):1227-1239. https://doi.org/10.1056/ NEJMra1005073

2. Cunha-Vaz J, Bernardes R, Lobo C (2011) Blood-retinal barrier. Eur J Ophthalmol 21(Suppl 6):S3-S9

3. Speiser P, Gittelsohn AM, Patz A (1968) Studies on diabetic retinopathy. 3. Influence of diabetes on intramural pericytes. Arch Ophthalmol 80(3):332-337. https://doi.org/10.1001/archopht. 1968.00980050334007

4. Leal EC, Martins J, Voabil P et al (2010) Calcium dobesilate inhibits the alterations in tight junction proteins and leukocyte 
adhesion to retinal endothelial cells induced by diabetes. Diabetes 59(10):2637-2645. https://doi.org/10.2337/db09-1421

5. Navaratna D, McGuire PG, Menicucci G, Das A (2007) Proteolytic degradation of VE-cadherin alters the blood-retinal barrier in diabetes. Diabetes 56(9):2380-2387. https://doi.org/10.2337/db061694

6. Roy S, Ha J, Trudeau K, Beglova E (2010) Vascular basement membrane thickening in diabetic retinopathy. Curr Eye Res 35(12):1045-1056. https://doi.org/10.3109/02713683.2010. 514659

7. Semeraro F, Cancarini A, dell'Omo R, Rezzola S, Romano MR, Costagliola C (2015) Diabetic retinopathy: vascular and inflammatory disease. J Diabetes Res 2015:582060

8. Zeng HY, Green WR, Tso MO (2008) Microglial activation in human diabetic retinopathy. Arch Ophthalmol 126(2):227-232. https://doi.org/10.1001/archophthalmol.2007.65

9. Aiello LP, Avery RL, Arrigg PG et al (1994) Vascular endothelial growth factor in ocular fluid of patients with diabetic retinopathy and other retinal disorders. N Engl J Med 331(22):1480-1487. https://doi.org/10.1056/NEJM199412013312203

10. Gavard J, Gutkind JS (2006) VEGF controls endothelial-cell permeability by promoting the beta-arrestin-dependent endocytosis of VE-cadherin. Nat Cell Biol 8(11):1223-1234. https://doi.org/10. 1038/ncb1486

11. Sidibe A, Imhof BA (2014) VE-cadherin phosphorylation decides: vascular permeability or diapedesis. Nat Immunol 15(3):215-217. https://doi.org/10.1038/ni.2825

12. Aiello LP, Northrup JM, Keyt BA, Takagi H, Iwamoto MA (1995) Hypoxic regulation of vascular endothelial growth factor in retinal cells. Arch Ophthalmol 113(12):1538-1544. https://doi.org/10. 1001/archopht.1995.01100120068012

13. Das A, Stroud S, Mehta A, Rangasamy S (2015) New treatments for diabetic retinopathy. Diabetes Obes Metab 17(3):219-230. https://doi.org/10.1111/dom.12384

14. Lampugnani MG, Orsenigo F, Gagliani MC, Tacchetti C, Dejana E (2006) Vascular endothelial cadherin controls VEGFR-2 internalization and signaling from intracellular compartments. J Cell Biol 174(4):593-604. https://doi.org/10.1083/jcb.200602080

15. Davidson MK, Russ PK, Glick GG, Hoffman LH, Chang MS, Haselton FR (2000) Reduced expression of the adherens junction protein cadherin-5 in a diabetic retina. Am J Ophthalmol 129(2): 267-269. https://doi.org/10.1016/S0002-9394(99)00323-2

16. Yan Y, Chang Q, Li Q et al (2015) Identification of plasma vascular endothelia-cadherin as a biomarker for coronary artery disease in type 2 diabetes mellitus patients. Int J Clin Exp Med 8(10):1946619470

17. Lee CS, Kim YG, Cho HJ et al (2016) Dipeptidyl peptidase-4 inhibitor increases vascular leakage in retina through VE-cadherin phosphorylation. Sci Rep 6(1):29393. https://doi.org/10.1038/ srep29393

18. He J, Wang H, Liu Y, Li W, Kim D, Huang H (2015) Blockade of vascular endothelial growth factor receptor 1 prevents inflammation and vascular leakage in diabetic retinopathy. J Ophthalmol 2015: 605946

19. Young JA, Ting KK, Li J et al (2013) Regulation of vascular leak and recovery from ischemic injury by general and VE-cadherinrestricted miRNA antagonists of miR-27. Blood 122(16):29112919. https://doi.org/10.1182/blood-2012-12-473017

20. Zhao Y, Ting KK, Li J et al (2017) Targeting vascular endothelialcadherin in tumor-associated blood vessels promotes $\mathrm{T}$ cellmediated immunotherapy. Cancer Res 77(16):4434-4447. https:// doi.org/10.1158/0008-5472.CAN-16-3129

21. Zhou Q, Gallagher R, Ufret-Vincenty R, Li X, Olson EN, Wang S (2011) Regulation of angiogenesis and choroidal neovascularization by members of microRNA-23 27 24 clusters. Proc Natl
Acad Sci U S A 108(20):8287-8292. https://doi.org/10.1073/ pnas. 1105254108

22. Nielsen LB, Wang C, Sorensen K et al (2012) Circulating levels of microRNA from children with newly diagnosed type 1 diabetes and healthy controls: evidence that miR-25 associates to residual betacell function and glycaemic control during disease progression. Exp Diabetes Res 2012:896362

23. Karolina DS, Tavintharan S, Armugam A et al (2012) Circulating miRNA profiles in patients with metabolic syndrome. J Clin Endocrinol Metab 97(12):E2271-E2276. https:/doi.org/10.1210/ jc.2012-1996

24. Shen W, Fruttiger M, Zhu L et al (2012) Conditional Muller cell ablation causes independent neuronal and vascular pathologies in a novel transgenic model. J Neurosci 32(45):15715-15727. https:// doi.org/10.1523/JNEUROSCI.2841-12.2012

25. Smith LE, Wesolowski E, McLellan A et al (1994) Oxygen-induced retinopathy in the mouse. Invest Ophthalmol Vis Sci 35(1):101-111

26. Lange C, Ehlken C, Stahl A, Martin G, Hansen L, Agostini HT (2009) Kinetics of retinal vaso-obliteration and neovascularisation in the oxygen-induced retinopathy (OIR) mouse model. Graefes Arch Clin Exp Ophthalmol 247(9):1205-1211. https://doi.org/10. 1007/s00417-009-1116-4

27. Sasongko MB, Wong TY, Nguyen TT, Cheung CY, Shaw JE, Wang JJ (2011) Retinal vascular tortuosity in persons with diabetes and diabetic retinopathy. Diabetologia 54(9):2409-2416. https://doi. org/10.1007/s00125-011-2200-y

28. Hammes HP, Lin J, Renner O et al (2002) Pericytes and the pathogenesis of diabetic retinopathy. Diabetes 51(10):3107-3112. https://doi.org/10.2337/diabetes.51.10.3107

29. Robinson R, Barathi VA, Chaurasia SS, Wong TY, Kern TS (2012) Update on animal models of diabetic retinopathy: from molecular approaches to mice and higher mammals. Dis Model Mech 5(4): 444-456. https://doi.org/10.1242/dmm.009597

30. Gerhardinger C, Dagher Z, Sebastiani P, Park YS, Lorenzi M (2009) The transforming growth factor-beta pathway is a common target of drugs that prevent experimental diabetic retinopathy. Diabetes 58(7):1659-1667. https://doi.org/10.2337/db08-1008

31. Chen Y, Hu Y, Zhou T et al (2009) Activation of the Wnt pathway plays a pathogenic role in diabetic retinopathy in humans and animal models. Am J Pathol 175(6):2676-2685. https://doi.org/10. 2353/ajpath.2009.080945

32. Gerhardt H, Betsholtz C (2003) Endothelial-pericyte interactions in angiogenesis. Cell Tissue Res 314(1):15-23. https://doi.org/10. 1007/s00441-003-0745-x

33. Bentley K, Franco CA, Philippides A et al (2014) The role of differential VE-cadherin dynamics in cell rearrangement during angiogenesis. Nat Cell Biol 16(4):309-321. https://doi.org/10.1038/ ncb2926

34. Taddei A, Giampietro C, Conti A et al (2008) Endothelial adherens junctions control tight junctions by VE-cadherin-mediated upregulation of claudin-5. Nat Cell Biol 10(8):923-934. https://doi.org/10. $1038 /$ ncb1752

35. Luo Y, Xiao W, Zhu X et al (2011) Differential expression of claudins in retinas during normal development and the angiogenesis of oxygen-induced retinopathy. Invest Ophthalmol Vis Sci 52(10):7556-7564. https://doi.org/10.1167/iovs.11-7185

36. Dalkara T, Gursoy-Ozdemir Y, Yemisci M (2011) Brain microvascular pericytes in health and disease. Acta Neuropathol 122(1):1-9. https://doi.org/10.1007/s00401-011-0847-6

37. McGuire PG, Rangasamy S, Maestas J, Das A (2011) Pericytederived sphingosine 1-phosphate induces the expression of adhesion proteins and modulates the retinal endothelial cell barrier. Arterioscler Thromb Vasc Biol 31:e107-e115

38. Cunha SI, Pietras K (2011) ALK1 as an emerging target for antiangiogenic therapy of cancer. Blood 117(26):6999-7006. https://doi.org/10.1182/blood-2011-01-330142 
39. Rudini N, Felici A, Giampietro C et al (2008) VE-cadherin is a critical endothelial regulator of TGF-beta signalling. EMBO J 27(7):993-1004. https://doi.org/10.1038/emboj. 2008.46

40. Li Q, Verma A, Han PY et al (2010) Diabetic eNOS-knockout mice develop accelerated retinopathy. Invest Ophthalmol Vis Sci 51(10): 5240-5246. https://doi.org/10.1167/iovs.09-5147

41. Fu S, Dong S, Zhu M et al (2015) Muller glia are a major cellular source of survival signals for retinal neurons in diabetes. Diabetes 64(10):3554-3563. https://doi.org/10.2337/db15-0180

42. Hnik P, Boyer DS, Grillone LR, Clement JG, Henry SP, Green EA (2009) Antisense oligonucleotide therapy in diabetic retinopathy. J Diabetes Sci Technol 3(4):924-930. https://doi.org/10.1177/ 193229680900300440

43. Omri S, Behar-Cohen F, de Kozak Y et al (2011) Microglia/ macrophages migrate through retinal epithelium barrier by a transcellular route in diabetic retinopathy: role of $\mathrm{PKC} \zeta$ in the Goto Kakizaki rat model. Am J Pathol 179(2):942-953. https://doi.org/ 10.1016/j.ajpath.2011.04.018

44. Krady JK, Basu A, Allen CM et al (2005) Minocycline reduces proinflammatory cytokine expression, microglial activation, and caspase- 3 activation in a rodent model of diabetic retinopathy.
Diabetes 54(5):1559-1565. https://doi.org/10.2337/diabetes.54.5. 1559

45. da Fonseca AC, Matias D, Garcia C et al (2014) The impact of microglial activation on blood-brain barrier in brain diseases. Front Cell Neurosci 8:362

46. Ryu JK, McLarnon JG (2009) A leaky blood-brain barrier, fibrinogen infiltration and microglial reactivity in inflamed Alzheimer's disease brain. J Cell Mol Med 13(9a):2911-2925. https://doi.org/ 10.1111/j.1582-4934.2008.00434.x

47. Monickaraj F, McGuire PG, Nitta CF, Ghosh K, Das A (2016) Cathepsin D: an M $\phi$-derived factor mediating increased endothelial cell permeability with implications for alteration of the bloodretinal barrier in diabetic retinopathy. FASEB J 30(4):1670-1682. https://doi.org/10.1096/fj.15-279802

48. Deliyanti D, Talia DM, Zhu T et al (2017) Foxp3(+) Tregs are recruited to the retina to repair pathological angiogenesis. Nat Commun 8(1):748. https://doi.org/10.1038/s41467-017-00751-w

49. Amadio M, Govoni S, Pascale A (2016) Targeting VEGF in eye neovascularization: What's new? A comprehensive review on current therapies and oligonucleotide-based interventions under development. Pharmacol Res 103:253-269. https://doi.org/10.1016/j. phrs.2015.11.027 